\title{
Case report: CLINICAL COURSE AND TREATMENT OF ANTI- HMGCR ANTIBODY-ASSOCIATED NECROTIZING AUTOIMMUNE MYOPATHY
}

Omer Hussain, Aadil Al Ghafri, Trevor Duffy, Eithne Murphy, Maurice Barry

Connolly Hospital, Dublin, Ireland

\section{Objective}

We report a clinical case of necrotizing autoimmune myopathy (NAM) associated with autoantibody against 3-hydroxy-3-methylglutaryl-coenzyme A reductase (HMGCR) and describe the challenges in clinical and therapeutic strategies of managing this patient.

\section{Methods}

We describe a case of immune-mediated necrotizing myopathy (Clinical, laboratory, EMG, and histopathologic results and response to immunomodulation therapy) associated with station exposure history and positive HMGCR antibodies.

\section{$\underline{\text { Results }}$}

This patient presented with proximal and distal myopathies with history of statin exposure with persistent symptoms post statin cessation. CPK ranged from 20000 to 35000 IU/L. EMG was consistent with an inflammatory myopathic disease. Muscle biopsies revealed a pauci-immune necrotizing myopathy. Improvement in clinical symptomatology and muscle enzymes shown with steroids and other immunomodulators including Methotrexate, IVIG and Rituximab.

\section{Conclusion}

HMGCR antibodies associated Necrotizing autoimmune myopathies is recognized myopathic disease with challenging therapeutic strategies. 